\title{
ON $q$-HYPERELLIPTIC $k$-BORDERED TORI
}

\author{
B. ESTRADA and E. MARTÍNEZ \\ Departamento de Matemáticas Fundamentales, UNED, Senda del Rey s/n, 28040-Madrid, Spain \\ e-mail:bestra@mat.uned.es,emartinez@mat.uned.es
}

(Received 14 December, 1999)

\begin{abstract}
A compact Klein surface $X$ is a compact surface with a dianalytic structure. Such a surface is said to be $q$-hyperelliptic if it admits an involution $\phi$, that is an order two automorphism, such that $X /\langle\phi\rangle$ has algebraic genus $q$. A Klein surface of genus 1 and $k$ boundary components is a $k$-bordered torus.

By means of NEC groups, $q$-hyperelliptic $k$-bordered tori are studied and a geometrical description of their associated Teichmüller spaces is given.
\end{abstract}

1991 Mathematics Subject Classification. 30F50.

1. Introduction. Klein surfaces, introduced from a modern point of view by Alling and Greenleaf [1], are surfaces endowed with a dianalytic structure. A compact orientable Klein surface $X$ with topological genus 1 and $k \geq 1$ boundary components is a $k$-bordered torus. The surface $X$ is said to be $q$-hyperelliptic if and only if $X$ admits an involution $\phi$, that is an order two automorphism, such that $X /\langle\phi\rangle$ is an orbifold with algebraic genus $q$. In the particular cases $q=0,1, X$ is hyperelliptic and elliptic-hyperelliptic respectively.

Non-Euclidean crystallographic groups (NEC groups in short) where introduced by Wilkie [16] and Macbeath [10], and they are an important tool in the study of Klein surfaces since the results of Preston [14] and May [13]. Klein surfaces can be seen as quotients of the hyperbolic plane under the action of an NEC group. In particular, when $X$ is a torus, then $X=\mathcal{D} / \Gamma$, where $\mathcal{D}$ denotes the hyperbolic plane and $\Gamma$ is a surface NEC group with signature:

$$
\sigma(\Gamma)=\left(1,+,[-],\left\{(-)^{k}\right\}\right), \quad k \geq 1 .
$$

The surface $X$ is $q$-hyperelliptic if and only if there exists an NEC group $\Gamma_{1}$ with $\Gamma \triangleleft_{2} \Gamma_{1}$ such that $\Gamma_{1} / \Gamma=\langle\phi\rangle$. If $k>4 q$ the group $\Gamma_{1}$ is unique [2] and $\Gamma_{1}$ is said to be the q-hyperellipticity group. In this case the automorphism $\phi$ is central in the group $\operatorname{Aut}(X)$ and it is called the $q$-hyperelliptic involution. The $q$-hyperelliptic surfaces have been studied in [2], [3], [5], and [8].

The aim of this work is the geometrical study of the Teichmüller space of $q$ hyperelliptic tori by means of fundamental regions of NEC groups. This technique was used in [6] and for the Moduli space of Riemann surfaces in [7].

In the next Section we give the necessary preliminaries about NEC groups and Klein surfaces. In Section 3 the signature of the $q$-hyperelliptic group $\Gamma_{1}$ is obtained. As a result $\Gamma_{1}$ may belong to one of four different classes. Afterwards we construct

${ }^{1}$ Partially supported by DGICYT PB98-0017 
fundamental regions $R$ with all right angles for groups belonging to the above classes. The parameters (lengths of the sides in $R$ ) can be taken as coordinates in the Teichmüller space of $q$-hyperelliptic tori. It is done in Section 4.

2. Preliminaries on NEC groups. An NEC group $\Gamma$ is a discrete subgroup of isometries of the hyperbolic plane $\mathcal{D}$, including reversing-orientation elements, with compact quotient $X=\mathcal{D} / \Gamma$.

Each NEC group $\Gamma$ is given a signature [10]

$$
\sigma(\Gamma)=\left(g, \pm,\left[m_{1}, \ldots, m_{r}\right],\left\{\left(n_{i 1}, \ldots, n_{i s_{i}}\right), i=1, \ldots, k\right\}\right)
$$

where $g, m_{i}, n_{i j}$ are integers verifying $g \geq 0, m_{i} \geq 2, n_{i j} \geq 2 . g$ is the topological genus of $X$. The sign determines the orientability of $X$. The numbers $m_{i}$ are the proper periods corresponding to cone points in $X$. The brackets $\left(n_{i 1}, \ldots, n_{i s_{i}}\right)$ are the period-cycles. The number $k$ of period-cycles is equal to the number of boundary components of $X$. Numbers $n_{i j}$ are the periods of the period-cycle $\left(n_{i 1}, \ldots, n_{i s_{i}}\right)$ also called link-periods, corresponding to corner points in the boundary of $X$. The number $p=\eta g+k-1$, where $\eta=1$ or 2 if the sign of $\sigma(\Gamma)$ is ' - ' or ' + ' respectively, is called the algebraic genus of $X$.

The signature determines a presentation $[\mathbf{1 0}]$ of $\Gamma$ :

Generators

$\begin{array}{ll}x_{i} & i=1, \ldots, r ; \\ e_{i} & i=1, \ldots, k ; \\ c_{i, j} & i=1, \ldots, r ; \quad j=0, \ldots, s_{i} ; \\ a_{i}, b_{i} & i=1, \ldots, g ; \text { (if } \sigma \text { has sign '+'); } \\ d_{i} & i=1, \ldots, g . \quad \text { (if } \sigma \text { has sign '-'). }\end{array}$

Relations:

$$
\begin{array}{ll}
x_{i}^{m_{i}}=1 ; & i=1, \ldots, r ; \\
c_{i, j-1}^{2}=c_{i, j}^{2}=\left(c_{i, j-1} c_{i, j}\right)^{n_{i, j}}=1 ; & i=1, \ldots, k ; j=1, \ldots, s_{i} ; \\
e_{i}^{-1} c_{i, 0} e_{i} c_{i, s_{i}}=1 ; & i=1, \ldots, k ; \\
\prod_{i=1}^{r} x_{i} \prod_{i=1}^{k} e_{i} \prod_{i=1}^{g}\left(a_{i} b_{i} a_{i}^{-1} b_{i}^{-1}\right)=1 ; & i=1, \ldots, g ; \quad \text { (if } \sigma \text { has sign '+'); } \\
\prod_{i=1}^{r} x_{i} \prod_{i=1}^{k} e_{i} \prod_{i=1}^{g} d_{i}^{2}=1 ; & i=1, \ldots, g ; \text { (if } \sigma \text { has sign '-'); }
\end{array}
$$

The isometries $x_{i}$ are elliptic, $e_{i}, a_{i}, b_{i}$ are hyperbolic, $c_{i, j}$ are reflections and $d_{i}$ are glide reflections.

Wilkie in [16] found a fundamental region $R_{W}$ from which he obtained the algebraic structure of NEC groups. The region $R_{W}$ is called a canonical region or Wilkie region.

For an NEC group $\Gamma$ with signature as (2.1) the region $R_{W}$ is a hyperbolic polygon with sides labelled in anticlockwise order as follows

$$
\varepsilon_{1}, \gamma_{10}, \ldots, \gamma_{1 s_{1}}, \varepsilon_{1}^{\prime}, \ldots, \varepsilon_{k}, \gamma_{k 0}, \ldots, \gamma_{k s_{k}}, \varepsilon_{k}^{\prime}, \alpha_{1}, \beta_{1}^{\prime}, \alpha_{1}^{\prime}, \beta_{1}, \ldots, \alpha_{g}, \beta_{g}^{\prime}, \alpha_{g}^{\prime}, \beta_{g}
$$

if sign ' + ', or 


$$
\varepsilon_{1}, \gamma_{10}, \ldots, \gamma_{1 s_{1}}, \varepsilon_{1}^{\prime}, \ldots, \varepsilon_{k}, \gamma_{k 0}, \ldots, \gamma_{k s_{k}}, \varepsilon_{k}^{\prime}, \delta_{1}, \delta_{1}^{*}, \ldots, \delta_{g}, \delta_{g}^{*}
$$

if sign '-', where

$$
e_{i}\left(\varepsilon_{i}^{\prime}\right)=\varepsilon_{i}, \quad c_{i}\left(\gamma_{i}\right)=\gamma_{i}, \quad a_{i}\left(\alpha_{i}^{\prime}\right)=\alpha_{i}, \quad b_{i}\left(\beta_{i}^{\prime}\right)=\beta_{i}, \quad d_{i}\left(\delta_{i}^{*}\right)=\delta_{i} .
$$

Let us denote by $<s_{1}, s_{2}>$ the angle between two consecutive sides. In $R_{W}$ we have

$$
<\varepsilon_{i}, \gamma_{i}>=<\gamma_{i}, \varepsilon^{\prime}>=\pi / 2
$$

and the sum of the remaining angles (accidental cycle) is $2 \pi$. Without a loss of generality we may suppose $R_{W}$ is a convex polygon.

Every NEC group $\Gamma$ with signature (2.1) has associated to it a fundamental region whose area $\mu(\Gamma)$, called the area of the group (see [15]), is:

$$
\mu(\Gamma)=2 \pi\left(\eta g+k-2+\sum_{i=1}^{r}\left(1-\frac{1}{m_{i}}\right)+\frac{1}{2} \sum_{i=1}^{k} \sum_{j=1}^{s_{i}}\left(1-\frac{1}{n_{i, j}}\right)\right)
$$

An NEC group with signature (2.1) actually exists if and only if the right hand side of (2.2) is greater than 0 (see [17]).

If $\Gamma$ is a subgroup of an NEC group $\Gamma^{\prime}$ of finite index $N$, then $\Gamma$ is also an NEC group and the following Riemann-Hurwitz formula holds:

$$
\mu(\Gamma)=N \mu\left(\Gamma^{\prime}\right)
$$

Let $X$ be a Klein surface of topological genus $g$ with $k$ boundary components. Then by [14] there exists an NEC group $\Gamma$ with signature

$$
\sigma(\Gamma)=(g ; \pm ;[-],\{(-), . k .,(-)\})
$$

such that $X=\mathcal{D} / \Gamma$, where the sign is "+" if $X$ is orientable and "-" if not. An NEC group with signature (2.4) is called a surface group.

For each automorphism group $G$ of a surface $X=\mathcal{D} / \Gamma$ of algebraic genus $p>2$ there exists an NEC group $\Gamma^{\prime}$ such that $G=\Gamma^{\prime} / \Gamma$ where $\Gamma \subset \Gamma^{\prime} \subset N_{\mathcal{G}}$, and $N_{\mathcal{G}}$ denotes the normalizer of $\Gamma$ in the group $\mathcal{G}$, the full group of isometries of $\mathcal{D}$ [13].

We give two previous results from [2] in Proposition 2.1 for future reference.

Proposition 2.1. (a) The Klein surface $X=\mathcal{D} / \Gamma$ is q-hyperelliptic if and only if there exists an NEC group $\Gamma_{1}$ with algebraic genus $q$ such that $\Gamma \triangleleft_{2} \Gamma_{1}$.

(b) Let $X$ be a q-hyperelliptic Klein surface of algebraic genus $p \geq 2$ such that $p>4 q+1$. Then the group $\Gamma_{1}$ is unique and the automorphism $\phi,\left\langle\phi>=\Gamma_{1} / \Gamma\right.$, is central in $\operatorname{Aut}(X)$.

In our case the algebraic genus of $q$-hyperelliptic tori is $k+1$ so that the inequality in Proposition 2.1 (b) becomes $k>4 q$. 
3. The signature of the $\boldsymbol{q}$-hyperellipticity group $\Gamma_{1}$. Let $\Gamma$ be a surface NEC group with signature (1.1) and let $\Gamma_{1}$ be an NEC group with $\Gamma \triangleleft_{2} \Gamma_{1}$. Then the signature of $\Gamma_{1}$ is [2]:

$$
\sigma\left(\Gamma_{1}\right)=\left(g ; \pm ;\left[2^{r}\right],\left\{\left(2^{s_{1}}\right), \ldots,\left(2^{s_{n}}\right)\right\}\right)
$$

where $s_{i}$ are even and $q=\eta g+n-1$. We have denoted by $\left[2^{r}\right]$ the set of proper periods $[2, r ., 2]$, and in a similar way the link periods in the period-cycles.

Our first task is to look for the possible values for $g, r, n$ and $s_{i}$ in (3.1). This is done by means of (2.3). Let $m$ be the number of non empty period-cycles in (3.1).

Proposition 3.1. The actual values for $g, r$ and $m$ in (3.1) are given in the following table:

$\begin{array}{ccccc}\text { Case } & g & \eta & r & m \\ \text { I } & 0 & 2 & 0 & 0 \\ \text { II } & 0 & 2 & 0 & 1 \\ \text { III } & 0 & 2 & 0 & 2 \\ \text { IV } & 0 & 2 & 1 & 0 \\ \text { V } & 0 & 2 & 1 & 1 \\ \text { VI } & 0 & 2 & 2 & 0 \\ \text { VII } & 0 & 2 & 2 & 1 \\ \text { VIII } & 0 & 2 & 3 & 0 \\ \text { IX } & 0 & 2 & 4 & 0 \\ \text { X } & 1 & 1 & 0 & 0 \\ \text { XI } & 1 & 1 & 0 & 1 \\ \text { XII } & 1 & 1 & 1 & 0 \\ \text { XIII } & 1 & 1 & 2 & 0 \\ \text { XIV } & 1 & 2 & 0 & 0 \\ \text { XV } & 2 & 1 & 0 & 0\end{array}$

Proof. From (2.3) we have

$$
k=2\left(\eta g+n-2+\frac{r}{2}+\frac{1}{4} \sum_{i=1}^{n} s_{i}\right) .
$$

The number of non-empty period-cycles is $m$ and so

$$
k \leq 2(n-m)+\frac{1}{2} \sum_{i=1}^{n} s_{i}
$$

or, equivalently,

$$
-2 m \geq k-2 n-\frac{1}{4} \sum_{i=1}^{n} s_{i}
$$

From (3.2) we obtain

$$
k-2 n-\frac{1}{2} \sum_{i=1}^{n} s_{i}=2 \eta g-4+r
$$


and from (3.3) and (3.4)

$$
2 m \leq 4-(2 \eta g+r)
$$

Giving numeric values to $g$ and $r$ and taking account of the sign in the signature we obtain the entries of the table.

We are interested in the case when the group of the $q$-hyperellipticity is unique. As we saw in the previous section, $k$ must be greater than $4 q$; from now on we always suppose that this condition holds.

Theorem 3.2. Let $X=\mathcal{D} / \Gamma$ be a $\mathrm{k}$-bordered torus, $k>4 q$. Then $X$ is $q$-hyperelliptic if and only if there exists a unique NEC group $\Gamma_{1}$ with algebraic genus $q$, such that $\Gamma \triangleleft_{2} \Gamma_{1}$ and the signature of $\Gamma_{1}$ is one of the following four signatures:

(1) $\left(0,+,[-],\left\{(-)^{q},\left(2^{2(k-2 q+2)}\right)\right\}\right)$;

(2) $\left(0,+,[-],\left\{(-)^{q-1},\left(2^{s_{1}}\right),\left(2^{s_{2}}\right)\right\}\right)$, where $s_{1}+s_{2}=2(k-2 q+2), s_{1}$ and $s_{2}$ are even;

(3) $\left(0,+,\left[2^{4}\right],\{(-)\}\right)$, where $q=0, k=2$;

(4) $\left(1,-,[-],\left\{(-)^{q-1},\left(2^{2(k-2 q+2)}\right)\right\}\right)$.

Proof. First of all let us observe from (3.2) that if $m=0$ (every period-cycle is empty) then

$$
k=2(q-1)+r
$$

and in this case $k>4 q$ if and only if $r>2 q+2$. From Proposition 3.1 we have $r \leq 4$, then $m \neq 0$ except for the case IX.

Now we may discard a lot of cases in Proposition 3.1. The available ones are the cases II, III, V, VII, IX and XI. Each case gives us a possible signature for $\Gamma_{1}$ and for each one we must study the existence of an epimorphism

$$
\theta_{1}: \Gamma_{1} \longrightarrow Z_{2}=\{1, y\}
$$

with $\operatorname{ker} \theta_{1}=\Gamma$.

In order to construct such an epimorphism let us observe that since $\operatorname{ker} \theta_{1}$ must be an orientable surface group ([4, Chapter 2]),

(a) consecutive reflections in a period-cycle cannot have the same image by $\theta_{1}$,

(b) non orientable words (words in the generators of $\Gamma_{1}-\Gamma$ ) cannot belong to $\operatorname{ker} \theta_{1}$, and

(c) the image by $\theta_{1}$ of every elliptic generator must have order two.

Case II: $\sigma\left(\Gamma_{1}\right)=\left(0,+,[-],\left\{(-)^{n-1},\left(2^{s}\right)\right\}\right)$.

Since $\Gamma_{1}$ has algebraic genus $q$ then $n-1=q$. From (3.2) we have $k=2\left(n-2+\frac{s}{4}\right)$; therefore $s=2(k-2 q+2)$. 
To define $\theta_{1}$, we see (a) implies

$$
\begin{gathered}
\theta_{1}\left(c_{q+1,2 j}\right)=y, \quad j=0, \ldots, k-2 q+2 \\
\theta_{1}\left(c_{q+1,2 j+1}\right)=1, \quad j=0, \ldots, k-2 q+1 .
\end{gathered}
$$

Thus we obtain $k-2 q+2$ empty period-cycles in $\operatorname{ker} \theta_{1}$. The remaining $2(q-1)$ must be obtained from the empty period-cycles of $\Gamma_{1}: C_{1}, \ldots, C_{q}$. Then $q-1$ reflections from the set $\left\{c_{1,0}, \ldots, c_{q, 0}\right\}$ will be in $\operatorname{ker} \theta_{1}$, and for each one $\theta_{1}\left(e_{i}\right)=1$. Let us define

$$
\theta_{1}\left(c_{i, 0}\right)=\theta_{1}\left(e_{i}\right)=1
$$

for $i=1, \ldots, q-1$, and $\theta_{1}\left(c_{q, 0}\right)=1$. To complete the epimorphism there still are two images to determine: $\theta_{1}\left(e_{q}\right)$ and $\theta_{1}\left(e_{q+1}\right)$.

From the canonical relation $e_{1} \cdots e_{q+1}=1$, we have

$$
\theta_{1}\left(e_{q}\right)=\theta_{1}\left(e_{q+1}\right),
$$

and, by (b), $\theta_{1}\left(e_{q}\right)=1$; otherwise $e_{q} c_{q, 0}$ would be a non-orientable word in $\operatorname{ker} \theta_{1}$. Then

$$
\begin{aligned}
& \theta_{1}: \quad \Gamma_{1} \longrightarrow Z_{2} \\
& e_{i} \quad \longrightarrow \quad 1 \quad i=1, \ldots, q+1 \text {, } \\
& c_{i, 0} \quad \longrightarrow \quad 1 \quad i=1, \ldots, q-1 \text {, } \\
& c_{q, 0} \quad \longrightarrow \quad y \\
& c_{q+1,2 j} \longrightarrow y \\
& c_{q+1,2 j+1} \longrightarrow 1
\end{aligned}
$$

Furthermore, by construction, $\theta_{1}$ is unique up to automorphisms of $\Gamma_{1}$.

Case III: $\sigma\left(\Gamma_{1}\right)=\left(0,+,[-],\left\{(-)^{q-1},\left(2^{s_{1}}\right),\left(2^{s_{2}}\right)\right\}\right)$, with $s_{1}$ and $s_{2}$ even.

From (3.2) we have

$$
k=2\left(q-1+\frac{s_{1}+s_{2}}{4}\right)=2(q-1)+\frac{s_{1}+s_{2}}{2},
$$

and hence

$$
s_{1}+s_{2}=2(k-2 q+2) .
$$

Reasoning as in Case II we obtain the epimorphism $\theta_{1}$ (unique up to $\operatorname{Aut}\left(\Gamma_{1}\right)$ ) defined as follows:

$$
\begin{aligned}
& \theta_{1}: \Gamma_{1} \longrightarrow Z_{2} \\
& e_{i} \longrightarrow 1 \quad i=1, \ldots, q+1, \\
& c_{i, 0} \quad \longrightarrow \quad 1 \quad i=1, \ldots, q-1, \\
& c_{i, j} \longrightarrow y \quad i=q, q+1, j \text { even, } \\
& c_{i, j} \longrightarrow 1 \quad i=q, q+1, j \text { odd. }
\end{aligned}
$$


Case V: $\sigma\left(\Gamma_{1}\right)=\left(0,+,[2],\left\{(-)^{q},\left(2^{s}\right)\right\}\right)$.

From the relation (3.2) we obtain

$$
k=2\left(q-1+\frac{1}{2}+\frac{s}{2}\right)=2 q-1+\frac{s}{2},
$$

and hence

$$
s=2(k-2 q+2) .
$$

But the number of period-cycles in $\operatorname{ker} \theta_{1}$ is

$$
2 l+(k-2 q+1)
$$

where $l=\#\left\{c_{i, 0}: \theta_{1}\left(c_{i, 0}\right)=1, i=1, \ldots, q\right\}$. This number never equals $k$. So this case must be discarded.

Case VII: $\sigma\left(\Gamma_{1}\right)=\left(0,+,[2,2],\left\{(-)^{q},\left(2^{s}\right)\right\}\right)$.

Every epimorphism $\theta_{1}: \Gamma_{1} \longrightarrow Z_{2}$ such that $\operatorname{ker} \theta_{1}$ is a surface group must satisfy

$$
\theta_{1}\left(x_{1}\right)=\theta_{1}\left(x_{2}\right)=\theta_{1}\left(c_{i, j}\right)=y
$$

for some $c_{i, j} \in \Gamma_{1}$. Then $\operatorname{ker} \theta_{1}$ is non-orientable, and this case must also be discarded.

Case IX: $\sigma\left(\Gamma_{1}\right)=\left(0,+,\left[2^{4}\right],\{(-)\}\right)$.

In this case the epimorphism $\theta_{1}: \Gamma_{1} \longrightarrow Z_{2}$ such that $\operatorname{ker} \theta_{1}$ is a torus with two boundaries is defined by

$$
\begin{aligned}
& \theta_{1}: \Gamma_{1} \longrightarrow Z_{2} \\
& x_{i} \quad \longrightarrow \quad y \quad i=1, \ldots, 4, \quad(\operatorname{see}(\mathrm{c})) \\
& e_{1} \longrightarrow 1 \\
& c_{1} \longrightarrow 1
\end{aligned}
$$

is unique up to $\operatorname{Aut}\left(\Gamma_{1}\right)$.

Case XI: $\sigma\left(\Gamma_{1}\right)=\left(1,-,[-],\left\{(-)^{q-1},\left(2^{s}\right)\right\}\right)$.

From $(3.2) k=2(q-1)+\frac{s}{2}$. So that $s=2(k-2 q+2)$ and reasoning as in Case II, the epimorphism $\theta_{1}$ is defined by

$$
\begin{aligned}
& \theta_{1}: \quad \Gamma_{1} \longrightarrow Z_{2} \\
& d_{i} \longrightarrow y \\
& e_{i} \longrightarrow 1 \quad i=1, \ldots, q, \\
& c_{i, 0} \quad \longrightarrow \quad 1 \quad i=1, \ldots, q-1 \text {, } \\
& c_{q, 2 j} \longrightarrow y \\
& c_{q, 2 j+1} \quad \longrightarrow \quad 1
\end{aligned}
$$


4. Dimension of the Teichmüller space. In this Section we study the Teichmüller space associated to $q$-hyperelliptic $k$-bordered tori.

Let $\mathcal{G}$ be the full group of isometries of the hyperbolic plane $\mathcal{D}$. Given an NEC group $\Lambda$ let us denote by $\mathcal{R}(\Lambda, \mathcal{G})$ the set of monomorphisms $r: \Lambda \longrightarrow \mathcal{G}$ such that $r(\Lambda)$ is a discrete group and the quotient $\mathcal{D} / r(\Lambda)$ is compact. Two elements $r_{1}, r_{2} \in \mathcal{R}(\Lambda, \mathcal{G})$ are equivalent, $r_{1} \sim r_{2}$, if and only if there exists an element $g \in \mathcal{G}$ satisfying $r_{1}(\lambda)=g \quad r_{2}(\lambda) g^{-1}$, for every $\lambda \in \Lambda$ The quotient space $\mathcal{T}(\Lambda, \mathcal{G})=$ $\mathcal{R}(\Lambda, \mathcal{G}) / \sim$, the Teichmüller space of $\Lambda$, is homeomorphic to a cell with dimension $d(\Lambda)$. If $\Lambda$ is a Fuchsian group with (NEC) signature $\left(g,+,\left[m_{1}, \ldots m_{r}\right],\{-\}\right)$ it is well known that $d(\Lambda)=6 g+2 r-6$. It is proved in [15] that if $\Lambda$ is a proper NEC group then $d(\Lambda)=\frac{d\left(\Lambda^{+}\right)}{2}$.

The Teichmüller modular group $\mathcal{M}(\Lambda)$ of $\Lambda$ is the quotient $\operatorname{Aut}(\Lambda) / \operatorname{Inn}(\Lambda)[\mathbf{1 1}$, where $\operatorname{Aut}(\Lambda)$ is the full group of automorphisms of $\Lambda$ and we denote by $\operatorname{Inn}(\Lambda)$ the inner automorphisms.

Now let $\Gamma$ be an NEC group with signature $\left(1,+,[-],\left\{(-)^{k}\right\}\right)$ and $X=\mathcal{D} / \Gamma$. Let $\phi$ an automorphism of order two such that $X /\langle\phi\rangle=X_{1}$ has algebraic genus $q$ and let $\Gamma_{1}$ be an NEC group such that $X_{1}=\mathcal{D} / \Gamma_{1}$. We have seen in the previous section that if $k>4 q$ the group $\Gamma_{1}$ has a signature of four possible types. So we divide the $q$-hyperelliptic $k$-bordered tori into four classes according to whether the quotient by the $q$-hyperelliptic involution is:

(1) a sphere with corner points in a single connected boundary component;

(2) a sphere with corner points in two connected boundary components;

(3) a disc with four cone points;

(4) a non-orientable surface.

Hence the Teichmüller space

$$
\mathcal{T}_{q}=\{[r] \in \mathcal{T}: \mathcal{D} / r(\Gamma) \text { is a } q \text {-hyperelliptic } k \text {-bordered torus, } k>4 q\}
$$

becomes divided into four subspaces corresponding to the above classes:

$$
\mathcal{T}_{q}=\mathcal{T}_{q}^{1} \cup \mathcal{T}_{q}^{2} \cup \mathcal{T}_{q}^{3} \cup \mathcal{T}_{q}^{4}
$$

From [9] we have for $i=1,3$ and 4,

$$
\mathcal{T}_{q}^{i}=\bigcup_{\alpha \in \mathcal{M}(\Gamma)} \bar{\alpha}\left(\bigcup_{i_{\phi} \in \Phi\left(\Gamma, \Gamma_{1}, \Gamma_{1} / \Gamma\right)} i_{\phi}^{*}\left(\mathcal{T}\left(\Gamma_{1}, \mathcal{G}\right)\right)\right),
$$

where $\Gamma_{1}$ is the (unique) NEC group of the $q$-hyperellipticity of $X, \Phi\left(\Gamma, \Gamma_{1}, \Gamma_{1} / \Gamma\right)$ is the family of equivalence classes of surjections $\phi: \Gamma_{1} \longrightarrow Z_{2}$ with $\operatorname{ker} \phi=\Gamma$ modulo the action of $\operatorname{Aut}\left(\Gamma_{1}\right)$ and $\operatorname{Aut}\left(Z_{2}\right)$ and $i_{\phi}^{*}$ is the induced isometry by the inclusion $i_{\phi}: \operatorname{ker} \phi \longrightarrow \Gamma_{1}:$

$$
\begin{array}{rlll}
i_{\phi}^{*}: & \mathcal{T}\left(\Gamma_{1}\right) & \hookrightarrow & \mathcal{T}(\Gamma) \\
{[\tau]} & \longrightarrow & {\left[\tau i_{\phi}\right]}
\end{array}
$$

where $\tau \in \mathcal{R}(\Gamma, \mathcal{G})$.

In the class (2), we have a family of $q$-hyperellipticity groups, that will be denoted by $\Gamma_{1}^{S_{1}, s_{2}}$, with signature as in Theorem 3.2(2). Then, $\mathcal{T}_{q}^{2}$ is decomposed as follows: 


$$
\mathcal{T}_{q}^{2}=\bigcup_{s_{1}+s_{2}=2(k-2 q+2)} \mathcal{T}_{q}^{s_{1}, s_{2}},
$$

where $\mathcal{T}_{q}^{s_{1}, s_{2}}$ has the same expression as in (4.1), changing $\Gamma_{1}$ to $\Gamma_{1}^{s_{1}, s_{2}}$.

In all cases, the families $\Phi\left(\Gamma, \Gamma_{1}^{i}, \Gamma_{1}^{i} / \Gamma\right), i=1,3$ and 4 , and $\Phi\left(\Gamma, \Gamma_{1}^{s_{1}, s_{2}}, \Gamma_{1}^{s_{1}, s_{2}} / \Gamma\right)$ have a single element, as was shown in the proof of Theorem 3.2. There, we constructed the unique class of epimorphisms

$$
\phi: \Gamma_{1} \longrightarrow Z_{2}, \quad \operatorname{ker} \phi=\Gamma, \text { for all } q \text {-hyperellipticity groups } \Gamma_{1}
$$

(see Cases II, III, IX and XI).

So the conditions of Maclachlan's method [12, Lemma 3] hold. Thus, we may conclude that $\mathcal{T}_{q}^{i}$ is a submanifold of $\mathcal{T}(\Gamma)$ of dimension $d\left(\Gamma_{1}\right)=2 k-q-1$. We have proved the following Theorem.

THEOREM 4.1. The subspace of the Teichmüller space associated to each class of $q$ hyperelliptic $k$-bordered tori, with $k>4 q$, is a submanifold of dimension $2 k-q+1$.

5. Geometrical description of $\mathcal{T}$. The abstract concept of Teichmüller space $\mathcal{T}(\Gamma)$ of an NEC group $\Gamma$ can be interpreted by means of fundamental regions. As we have seen two elements $r_{1}, r_{2} \in \mathcal{R}(\Gamma)$ belong to the same class in $\mathcal{T}(\Gamma)$ if and only if there exists $g \in \mathcal{G}$ such that

$$
r_{1}(\gamma)=g r_{2}(\gamma) g^{-1}, \text { for all } \gamma \in \Gamma
$$

Equivalently, the fundamental regions of the NEC groups $r_{1}(\Gamma)$ and $r_{2}(\Gamma)$ are congruent, that is, there exists an isometry $g \in \mathcal{G}$ which transforms one of them on the other one. For this reason we can associate to each class in $\mathcal{T}(\Gamma)$ a normalized fundamental region $R$ such that the number of parameters involved in the construction of $R$ equals $d(\mathcal{T}(\Gamma))$, the dimension of the Teichmüller space.

Let $R_{1}$ be a fundamental region of the $q$-hyperellipticity group $\Gamma_{1}$. The canonical epimorphism $\theta_{1}: \Gamma_{1} \longrightarrow \Gamma_{1} / \Gamma$ gives us a way to obtain a fundamental region $R$ of $\Gamma$ from two copies of $R_{1}$. Our goal in this section will be the description of the necessary parameters in the construction of $R_{1}$. To do this we will transform a canonical Wilkie region $R_{W}$ into a right-angled fundamental region by a cutting and pasting procedure.

Description of $\mathcal{T}_{q}^{1}$. Let $\Gamma_{1}$ be the $q$-hyperellipticity group with signature

$$
\left(0,+,[-],\left\{(-)^{q},\left(2^{2(k-2 q+2)}\right)\right\}\right),
$$

and let $R_{W}$ be a Wilkie region of $\Gamma_{1}$ (see Figure 1).

Let us consider the following geodesics in $R_{W}$ : let $\lambda_{i}$ be the common orthogonal to $\gamma_{i}$ and $\gamma_{q+1,0},(i=1, \ldots, q)$. Every side $\gamma_{i}$ is divided by $\lambda_{i}$ in two pieces, $\gamma_{i}=\gamma_{i}^{1} \cup \gamma_{i}^{2}$, and $\gamma_{q+1,0}$ is divided by the $\lambda_{i}$ in $q+1$ pieces:

$$
\gamma_{q+1,0}=\bar{\gamma}_{0} \cup \ldots \cup \bar{\gamma}_{q}
$$




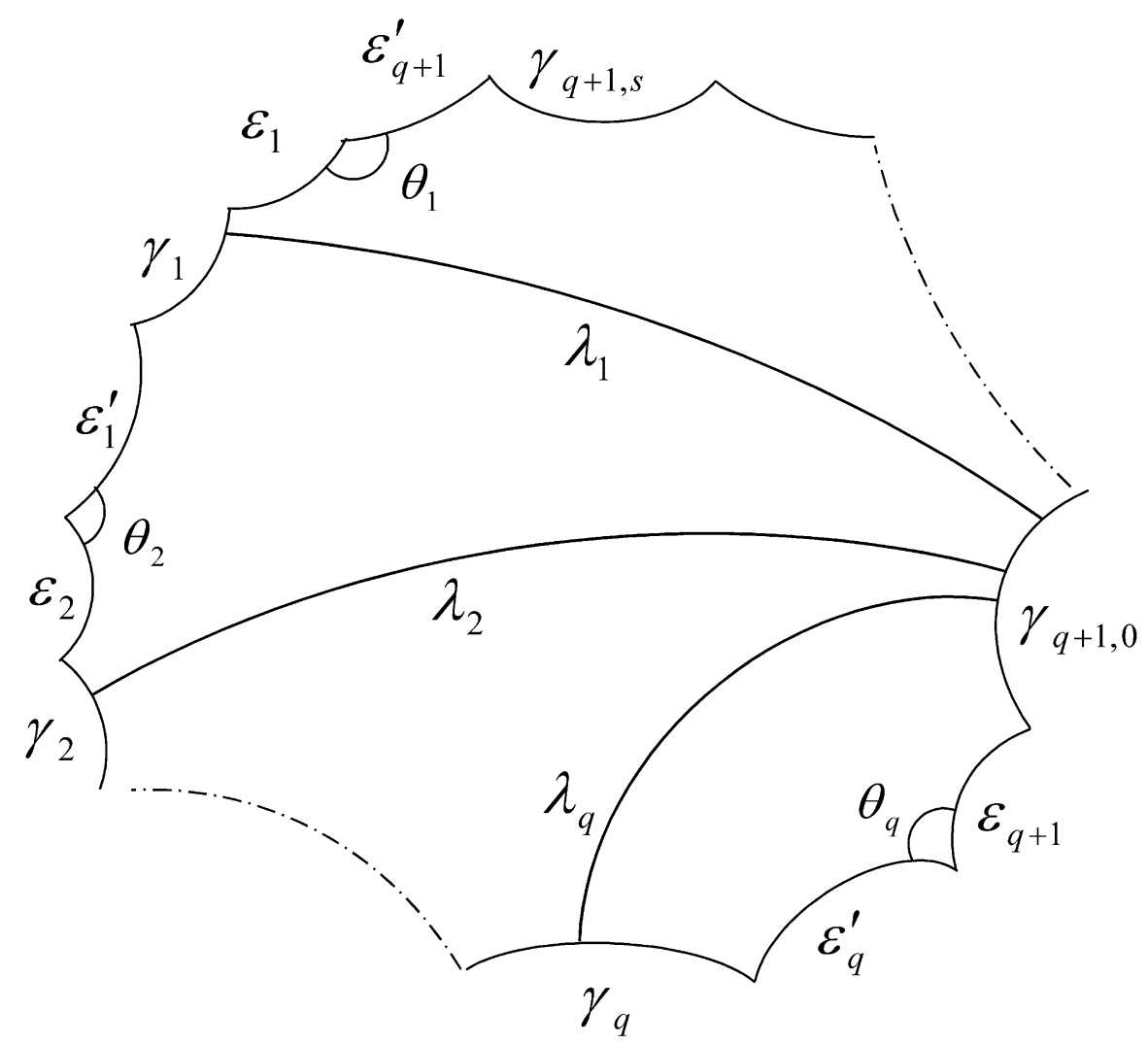

Figure 1. $R_{W}$.

Let us denote $R_{W}$ by $R_{q+1}$ and define transformations $Q_{\lambda_{i}}$ by the following rule: cut in $R_{i+1}$ by $\lambda_{i}$ the polygon which contains the side $\varepsilon_{i}^{\prime}$ and paste this side with $\varepsilon_{i}$ via $e_{i}$ to obtain $R_{i}$.

Then the region

$$
R^{*}=Q_{\lambda_{1}} \cdots Q_{\lambda_{q}}\left(R_{W}\right)
$$

is a right-angled fundamental region of $\Gamma_{1}$ with $2 k+4$ sides:

$$
\underbrace{\ldots, f_{i-1}\left(\bar{\gamma}_{i-1}\right), f_{i-1}\left(\lambda_{i}\right), f_{i-1}\left(\gamma_{i}^{*}\right), f_{i}\left(\lambda_{i}\right), \ldots}_{i=1, \ldots, q}, f_{q}\left(\bar{\gamma}_{q}\right) \cup \gamma_{q+1, s}, \gamma_{q+1, s-1}, \ldots, \gamma_{q+1,1},
$$

where $s=2(k-2 q+2)$ and

$$
\begin{aligned}
f_{0} & =i_{d}, \\
f_{i} & =e_{1} \cdots e_{i}, \\
\gamma_{i}^{*} & =e_{i}\left(\gamma_{i}^{2}\right) \cup \gamma_{i}^{1}, i=1, \ldots, q .
\end{aligned}
$$

The pairs of identified sides in $R^{*}$ are $\left(\lambda_{i}, f_{i}\left(\lambda_{i}\right)\right), i=1, \ldots, q$. 
Then we have constructed a hyperbolic right-angled polygon $R^{*}$ having $2 k+4$ sides and the $2 k+1$ consecutive sides with the following lengths:

$$
\left|\gamma_{q+1, s-3}\right|, \ldots,\left|\gamma_{q+1,1}\right|, \ldots, \underbrace{\left|\bar{\gamma}_{i-1}\right|,\left|\lambda_{i}\right|,\left|f_{i-1}\left(\gamma_{i}^{*}\right)\right|,\left|f_{i}\left(\lambda_{i}\right)\right|}_{i=0, \ldots, q} .
$$

Since $\left|f_{i-1}\left(\gamma_{i}^{*}\right)\right|=\left|\gamma_{i}\right|$ and $\left|f_{i}\left(\lambda_{i}\right)\right|=\left|\lambda_{i}\right|$ then we have the following free lengths $\left|\lambda_{1}\right|, \ldots,\left|\lambda_{q}\right|, \quad \quad(q$ orthogonal lines)

$\left|\gamma_{1}\right|, \ldots,\left|\gamma_{q}\right|, \quad \quad(q$ empty boundaries $)$

$\left|\gamma_{q+1,1}\right|, \ldots,\left|\gamma_{q+1, s-3}\right|, \quad(s-3$ sides of the non-empty $(q+1)$-boundary $)$

$\left|\bar{\gamma}_{0}\right|, \ldots,\left|\bar{\gamma}_{q-1}\right|, \quad \quad\left(q\right.$ pieces in that $\gamma_{q+1,0}$ becomes divided $)$.

Then, there are $2 k-q+1$ lengths and this number equals the dimension of $\mathcal{T}_{q}^{1}$.

Description of $\mathcal{T}_{q}^{s_{1}, s_{2}}$. Let $\Gamma_{1}$ be the $q$-hyperellipticity group with signature

$$
\left(0,+,[-],\left\{(-)^{q-1},\left(2^{s_{1}}\right),\left(2^{s_{2}}\right)\right\}\right)
$$

where $s_{1}, s_{2}$, are even positive integers such that $s_{1}+s_{2}=2(k-2 q+2)$; and let $R_{W}$ be a Wilkie region of $\Gamma_{1}$. To convert $R_{W}$ in a right-angled polygon, let us consider

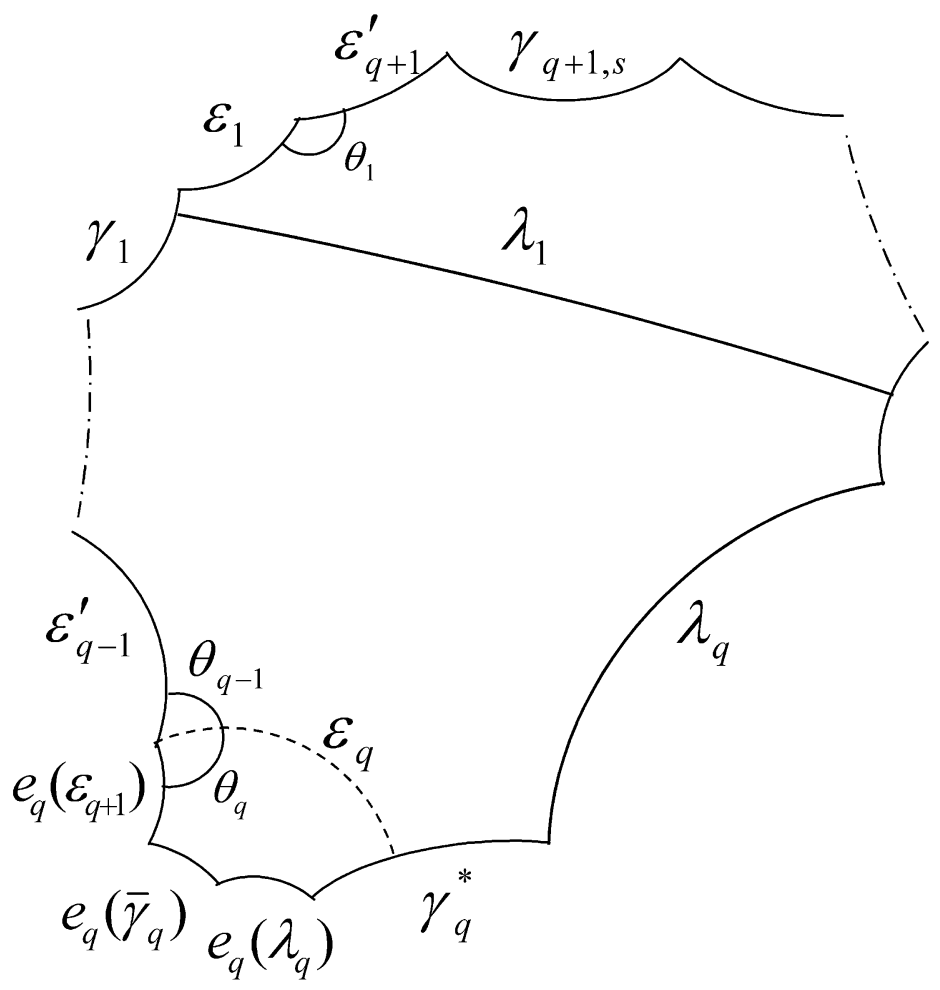

Figure 2. $Q_{\lambda_{q}}\left(R_{W}\right)$. 
the geodesics: $\lambda_{i},(i=1, \ldots, q-1)$ as in the description of $\mathcal{T}_{q}^{1}$, and $\lambda_{q}$ the common orthogonal to $\gamma_{q, 0}$ and $\gamma_{q+1,0}$.

Then the region

$$
R^{*}=Q_{\lambda_{1}} \cdots Q_{\lambda_{q}}\left(R_{W}\right)
$$

is a right-angled fundamental region of $\Gamma_{1}$ with $2 k+4$ sides. The perimeter of $R^{*}$, and the $2 k+1$ lengths of consecutive sides in $R^{*}$ are the same as (5.1) and (5.2), changing $s$ to $s_{2}$, and $f_{q-1}\left(\gamma_{q}^{*}\right)$ to $f_{q-1}\left(\gamma_{q, 0}^{*}\right) \cup f_{q}\left(\gamma_{q, 1}\right), \ldots, f_{q}\left(\gamma_{q, s_{1}}\right)$.

The involved lengths are:

$$
\begin{array}{ll}
\left|\lambda_{1}\right|, \ldots,\left|\lambda_{q}\right|, & (q \text { orthogonal lines }) \\
\left|\gamma_{1}\right|, \ldots,\left|\gamma_{q-1}\right|, & (q-1 \text { empty boundaries }) \\
\left|\gamma_{q, 0}\right|, \ldots,\left|\gamma_{q, s_{1}}\right|, & \left(s_{1}+1 \text { sides of the non-empty } q \text {-boundary }\right) \\
\left|\bar{\gamma}_{0}\right|, \ldots,\left|\bar{\gamma}_{q-1}\right|, & \left(q \text { pieces in that } \gamma_{q+1,0} \text { becomes divided }\right) \\
\left|\gamma_{q+1,1}\right|, \ldots,\left|\gamma_{q+1, s_{2}-3}\right|, & \left(s_{2}-3 \text { sides of the non-empty }(q+1) \text {-boundary }\right)
\end{array}
$$

Description of $\mathcal{T}_{q}^{3}$. Let $\Gamma_{1}$ be the $q$-hyperellipticity group with signature

$$
\left(0,+,\left[2^{4}\right],\{(-)\}\right)
$$

and let $R_{W}$ be a Wilkie region of $\Gamma_{1}$ (see Figure 3 ). The side-pairings are $\left(\delta_{i}^{\prime}, \delta_{i}\right)$ via the canonical generators $x_{i}, i=1, \ldots, 4$; and $\left(\varepsilon_{1}^{\prime}, \varepsilon_{1}\right)$ via $e_{i}$.

To convert $R_{W}$ in a right-angled polygon let us consider the orthogonal lines $\lambda_{i}$ from the vertex $X_{i}$ to $\gamma_{1}, i=1, \ldots, 4$. These geodesic segments divide $\gamma_{1}$ in five pieces $\bar{\gamma}_{i}, i=0, \ldots, 4$. Denote $R_{W}$ by $R_{4}$, and define the transformations $Q_{\lambda_{i}}$, $i=1, \ldots, 4$, as follows: cut in $R_{i}$ the polygon which contains the side $\delta_{i}^{\prime}$ and paste it with $\delta_{i}$ via $x_{i}$ to obtain $R_{i-1}$.

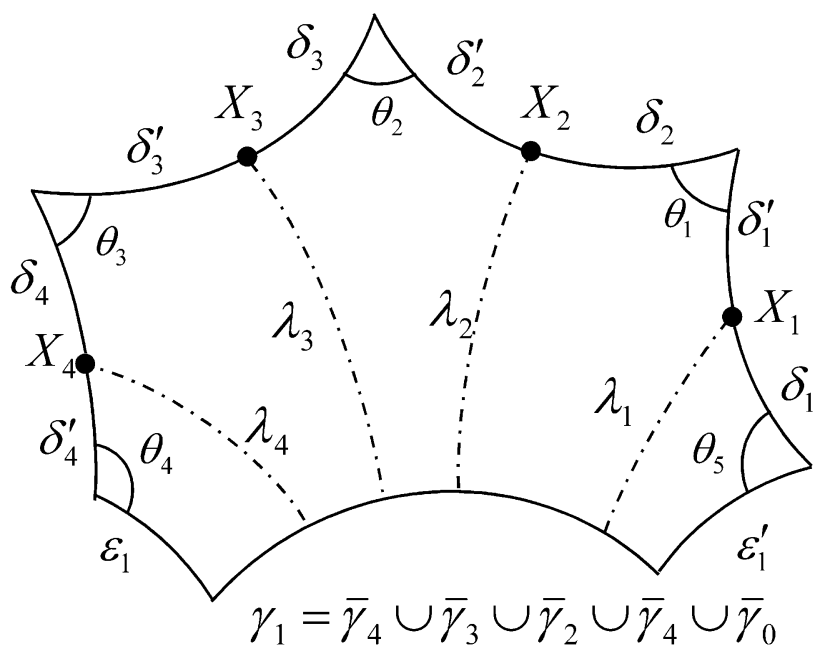

Figure 3. 


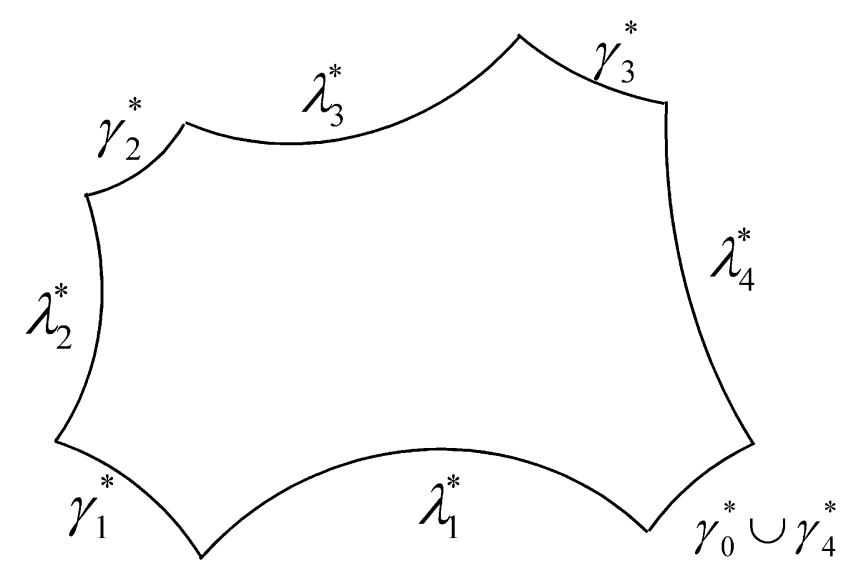

Figure 4. $R^{*}$. sides:

Then $R^{*}=Q_{\lambda_{1}} \circ \ldots \circ Q_{\lambda_{4}}\left(R_{W}\right)$ is a right-angled octagon with the following

$$
\gamma_{5}^{*} \cup \gamma_{1}^{*}, \lambda_{1}^{*}, \gamma_{2}^{*}, \lambda_{2}^{*}, \gamma_{3}^{*}, \lambda_{3}^{*}, \gamma_{4}^{*}, \lambda_{4}^{*},
$$

where

$$
\begin{aligned}
\lambda_{i}^{*} & =f_{i}\left(\lambda_{i}\right) \cup f_{i-1}\left(\lambda_{i}\right), \\
\gamma_{i}^{*} & =f_{i}\left(\bar{\gamma}_{i}\right), \\
f_{i} & =x_{1} i, \\
f_{0} & =i d .
\end{aligned}
$$

The polygon $R^{*}$ is completely determined by five lengths:

$$
2\left|\lambda_{1}\right|, \ldots, 2\left|\lambda_{4}\right|,\left|\bar{\gamma}_{1}\right|
$$

where $\left|\lambda_{i}\right|$ is the distance between the boundary and the cone point $X_{i}$, and $\left|\bar{\gamma}_{1}\right|$ is the distance between $\lambda_{1}$ and $\lambda_{2}$.

Description of $\mathcal{T}_{q}^{4}$. Let $\Gamma_{1}$ be the $q$-hyperellipticity group with signature

$$
\left(1,-,[-],\left\{(-)^{q-1},\left(2^{2(k-2 q+2)}\right)\right\}\right),
$$

and let $R_{W}$ be a Wilkie region of $\Gamma_{1}$ (see Figure 5).

Let $d$ be the glide reflection which transforms $\delta^{\prime}$ in $\delta$. The axis of $d$ is the geodesic joining the middle points of $\delta$ and $\delta^{\prime}$. Let $P, Q$, and $S$ be the vertices between the pair of sides $\left(\varepsilon_{q}^{\prime}, \delta\right),\left(\delta^{\prime}, \varepsilon_{1}\right)$ and $\left(\delta, \delta^{\prime}\right)$. Let us consider the following segments: $\varepsilon$ (respectively $\varepsilon^{\prime}$ ) the orthogonal to the axis of $d$ from $P$ (respectively $Q$ ) (see Figure 6). Then, $d^{2}$ is a hyperbolic transformation satisfying $d^{2}\left(\zeta^{\prime}\right)=\zeta$.

We are going to convert $R_{W}$ in a fundamental region of $\Gamma_{1}$ in which the sidepairings involves the hyperbolic transformation $d^{2}$. To do it, let us consider the geodesic $m$ orthogonal to the axis of $d$ which contains the vertex $S$, and the hyperbolic triangles $T_{1}$ and $T_{2}$ (see Figure 4). Then, the region 


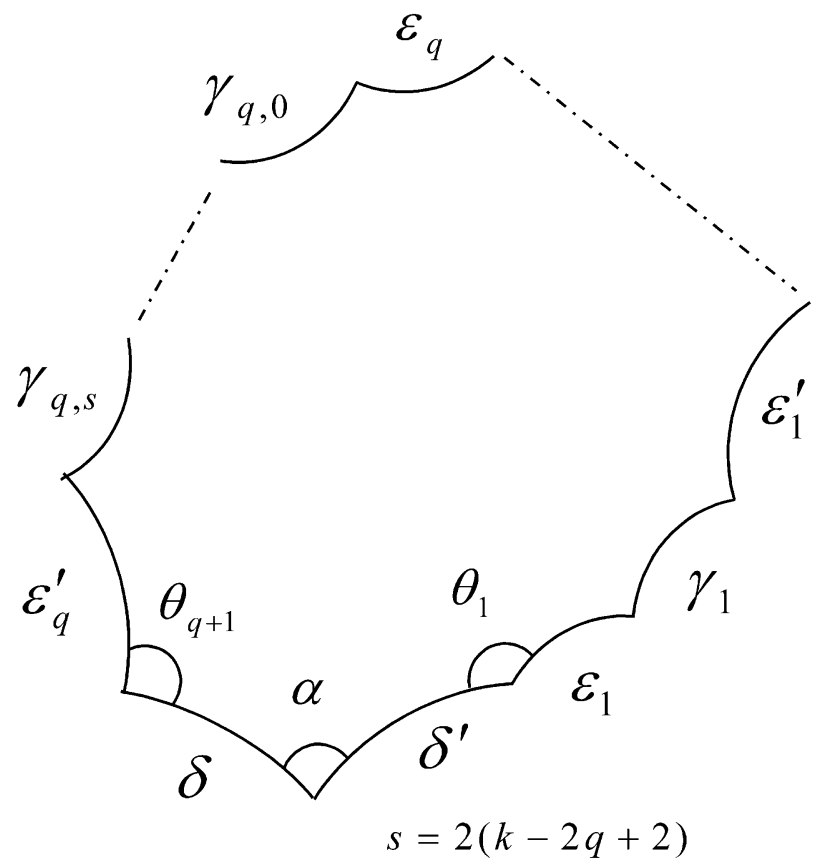

Figure 5 .

$$
\widehat{R}=R-\left(T_{1} \cup T_{2}\right) \cup d\left(T_{2}\right) \cup d^{-1}\left(T_{1}\right)
$$

is a fundamental region of $\Gamma_{1}$ that follows the pattern of identifications of $R$ in $\mathcal{T}_{q}^{1}$ (see Figure 7).

Then, we transform $\widehat{R}$ into a right-angled region as we did for $R$ in $\mathcal{T}_{q}^{1}$, obtaining in the same way the set of necessary lengths for its construction.

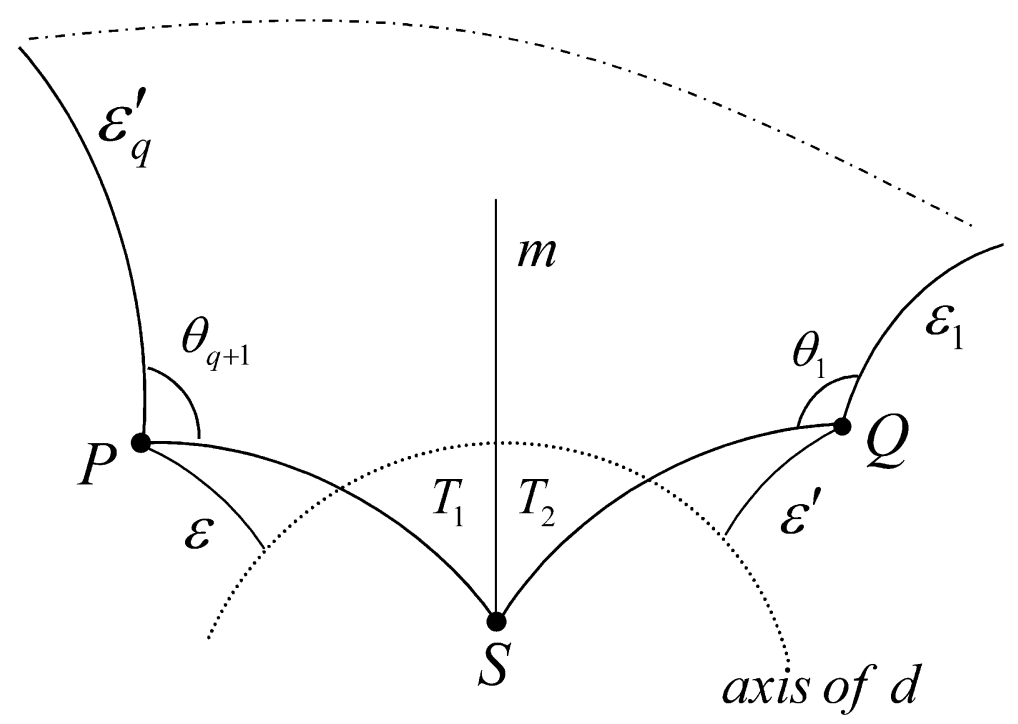

Figure 6. 


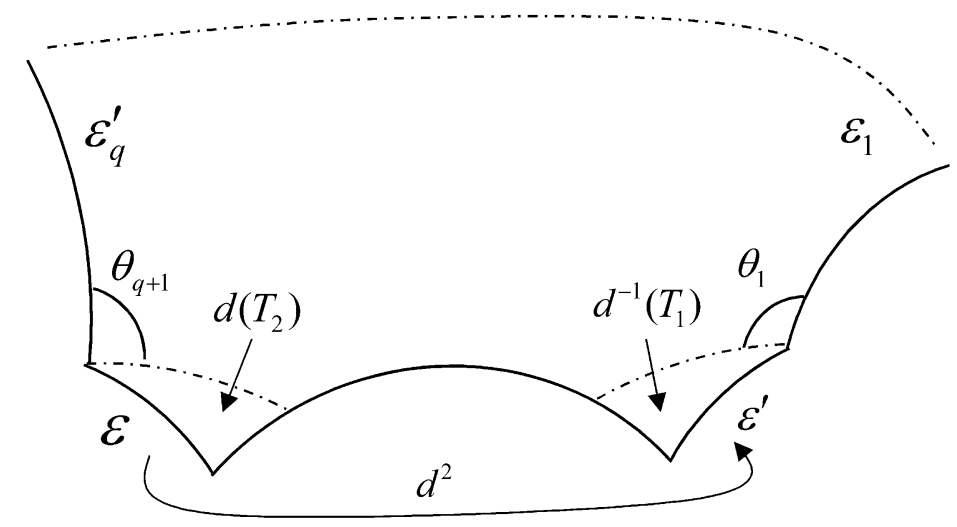

Figure 7.

\section{REFERENCES}

1. N. L. Alling and N. Greenleaf, Foundations of the theory of Klein surfaces, Lecture Notes in Mathematics 219 (Springer-Verlag, 1971).

2. E. Bujalance and J. J. Etayo, A characterization of $q$-Hyperelliptic compact planar Klein surfaces. Abh. Math. Sem. Univ. Hamburg 58 (1988), 95-104.

3. E. Bujalance, J. J. Etayo and J. M. Gamboa, Superficies de Klein elípticas hiperelípticas, Memorias de la Real Academia de Ciencias, XIX, (1985).

4. E. Bujalance, J. J. Etayo, J. M. Gamboa and G. Gromadzki. A combinatorial approach to automorphisms groups of compact bordered Klein surfaces, Lectures Notes in Mathematics 1439 (Springer-Verlag, 1990).

5. J. A. Bujalance and B. Estrada, Q-hyperelliptic compact non-orientable Klein surfaces without boundary, preprint.

6. A. F. Costa and E. Martínez, Planar hyperelliptic Klein surfaces and fundamental regions of N.E.C. groups. London Math Soc. Lecture Notes Series 173 (1992), 57-65.

7. A. F. Costa and E. Martínez, Parametrization of the Moduli Space of hyperelliptic and symmetric Riemann surfaces, Ann. Acad. Sci. Fenn. 22 (1997), 75-88.

8. B. Estrada, Automorphism groups of orientable elliptic-hyperelliptic Klein surfaces, Ann. Acad. Sci. Fenn. Math. 25 (2000), 439-456.

9. W. J. Harvey, On branch loci in Teichmüller space, Trans. Amer. Math. Soc. 153 (1971), 387-399.

10. A. M. Macbeath, The classification of non-Euclidean crystallographic groups. Canad. J. Math. 6 (1967), 1192-1205.

11. A. M. Macbeath and D. Singerman, Spaces of subgroups and Teichmüller space. Proc. London Math. Soc. (3) 31 (1975), 211-256.

12. C. Maclachlan, Smooth coverings of hyperelliptic surfaces. Quart. J. Math. Oxford Ser (2) 22 (1971), 117-123.

13. C. L. May, Large automorphism groups of compact Klein surfaces with boundary, Glasgow Math. J. 18 (1977), 1-10.

14. R. Preston, Projective structures and fundamental domains on compact Klein surfaces, Thesis, Univ. of Texas, (1975).

15. D. Singerman, On the structure of non-euclidean crystallographic groups. Proc. Cambridge Phil. Soc. 76 (1974), 233-240. 102.

16. H. C. Wilkie, On non-Euclidean Crystallographic groups, Math. Z. 91 (1966), 87-

17. H. Zieschang, E. Vogt and H. D. Coldewey, Surfaces and planar discontinuous groups, Lecture Notes in Mathematics, 835 (Springer-Verlag, 1980). 